\title{
FOLIATIONS BY MINIMAL SURFACES AND CONTACT STRUCTURES ON CERTAIN CLOSED 3-MANIFOLDS
}

\author{
RICHARD H. ESCOBALES JR.
}

Received 14 July 2002 and in revised form 7 August 2002

To Grant, Romana, Desmond, and Max

\begin{abstract}
Let $(M, g)$ be a closed, connected, oriented $C^{\infty}$ Riemannian 3-manifold with tangentially oriented flow $\mathbf{F}$. Suppose that $\mathbf{F}$ admits a basic transverse volume form $\mu$ and mean curvature one-form $\kappa$ which is horizontally closed. Let $\{X, Y\}$ be any pair of basic vector fields, so $\mu(X, Y)=1$. Suppose further that the globally defined vector $\mathscr{V}[X, Y]$ tangent to the flow satisfies $[Z, \mathscr{V}[X, Y]]=f_{Z} \mathscr{V}[X, Y]$ for any basic vector field $Z$ and for some function $f_{Z}$ depending on $Z$. Then, $\mathscr{V}[X, Y]$ is either always zero and $\mathbf{H}$, the distribution orthogonal to the flow in $T(M)$, is integrable with minimal leaves, or $\mathscr{V}[X, Y]$ never vanishes and $\mathbf{H}$ is a contact structure. If additionally, $M$ has a finite-fundamental group, then $\mathscr{V}[X, Y]$ never vanishes on $M$, by the above together with a theorem of Sullivan (1979). In this case $\mathbf{H}$ is always a contact structure. We conclude with some simple examples.
\end{abstract}

2000 Mathematics Subject Classification: 57R30, 53C25.

Throughout this paper, all maps, functions, and morphisms are assumed to be at least of class $C^{\infty}$. On a closed, connected, oriented $C^{\infty}$ Riemannian 3-manifold $(M, g)$, let $\mathbf{F}$ be a tangentially oriented flow. Let $\mathbf{V}$ denote the distribution tangent to the flow $\mathbf{F}$ and $\mathbf{H}$ the distribution orthogonal to $\mathbf{V}$ in $T M$ determined by the metric $g$. If $E$ is a vector field on $M, \mathscr{V} E$ and $\mathscr{H} E$ will denote the projections of $E$ onto the distributions $\mathbf{V}$ and $\mathbf{H}$, respectively. Call the vector field $E$ vertical if $\mathscr{V} E=E$. Call $E$ horizontal if $\mathscr{H} E=E$. Now, $\mathbf{F}$ can be viewed as a foliation of codimension 2 and leaf dimension 1 .

In general, a $C^{\infty}$ foliation of codimension $q$ on an $n$-dimensional manifold $M$ can be defined as a maximal family of $C^{\infty}$ submersions $f_{\alpha}: U_{\alpha} \rightarrow f_{\alpha}\left(U_{\alpha}\right) \subset \mathbb{R}^{q}$, where $\left\{U_{\alpha}\right\}_{\alpha \in \Lambda}$ is an open cover of $M$ and where, for each $\alpha, \beta \in \Lambda$ and each $x \in U_{\alpha} \cap U_{\beta}$, there exists a local diffeomorphism $\phi_{\beta \alpha}^{x}$ of $\mathbb{R}^{q}$, so $f_{\beta}=\phi_{\beta, \alpha}^{x} \circ f_{\alpha}$ in some neighborhood $U_{x}$ of $x$ (see [12, pages 2-3]). In the case of a flow $\mathbf{F}$, the codimension is $q=n-1$. In the setting of this paper, the submersions defining the flow $\mathbf{F}$ are $f_{\alpha}: U_{\alpha} \rightarrow f_{\alpha}\left(U_{\alpha}\right) \subset \mathbb{R}^{2}$.

A horizontal vector field $Z$ defined on some open set $U$, where $U \subset U_{\alpha}$, is called $f_{\alpha}$-basic provided $f_{\alpha *} Z$ is a well-defined vector field on $f_{\alpha}(U)$. As pointed out in [5] (for any metric $g$ ), if $U \subset U_{\beta}$, then $Z$ is also $f_{\beta}$-basic, so one can speak of $Z$ as a local basic vector field. We sometimes drop the word 
"local." Let $i(W)$ and $\theta(W)$ denote the interior product and the Lie derivative with respect to a vector field $W$. A differential form $\phi$ is called basic provided $i(W) \phi=0$ and $\theta(W) \phi=0$ for all vertical vector fields $W$ (see [16, page 118]). Throughout this paper, we will assume that $(M, g)$ admits a basic transverse volume element $\mu$ [16].

Let $D$ denote the Levi-Civita connection on $M$ and, following [6], we introduce the tensors $T$ and $A$ as follows. For vector fields $E$ and $F$ on $M$,

$$
\begin{aligned}
& T_{E} F=\mathscr{V} D_{\mathscr{V} E} \mathscr{H} F+\mathscr{H} D_{\mathscr{V} E} \mathscr{V} F, \\
& A_{E} F=\mathscr{V} D_{\mathscr{H} E} \mathscr{H} F+\mathscr{H} D_{\mathscr{H}} \mathscr{V} F .
\end{aligned}
$$

Then, $T$ and $A$ are tensors of type (1). These tensors satisfy the usual properties outlined in [6]. We note that if $X$ and $Y$ are horizontal,

$$
A_{X} Y \neq-A_{Y} X
$$

in general unless the flow $\mathbf{F}$ is bundle-like with respect to the metric $g$ (see [11, Lemma 1.2]) that is, if $X$ is a basic vector field, $W g(X, X)=0$ for every vertical vector field $W$. It is worth observing that if $\mathbf{F}$ is bundle-like with respect to $g$ and $\mathbf{H}$ is integrable, then the leaves of $\mathbf{H}$ are totally geodesic since, by (2), $A_{X} X=0$ for every horizontal $X$. This paper obtains conclusions without imposing the bundle-like assumption. For this reason, it is hoped the result will be of interest to topologists.

If $V$ is the unit vector tangent to the flow, we define the mean curvature one-form $\kappa$ as follows:

$$
\kappa(E)=g\left(E, T_{V} V\right)
$$

Call $\kappa$ horizontally closed if $d \kappa\left(Z_{1}, Z_{2}\right)=0$ for any horizontal fields $Z_{1}$ and $Z_{2}$. If a basic vector field $Z$ is defined on an open set $U$, the real-valued function $f_{Z}$, discussed in the theorem, will have $U$ as its domain as well. With these preparations, we give the following result.

THEOREM 1. Let $(M, g)$ be a closed, connected, oriented $C^{\infty}$ Riemannian 3manifold with tangentially oriented flow $\mathbf{F}$. Suppose the following conditions.

(a) The flow $\mathbf{F}$ admits a basic transverse volume form $\mu$.

(b) Its mean curvature one-form $\kappa$ is horizontally closed (or, less generally, $\kappa$ is basic).

Let $X$ and $Y$ be local basic vector fields on $M$ so $\mu(X, Y)=1$.

(c) The globally defined vector field $\mathscr{V}[X, Y]$ on $M$ satisfies

$$
[Z, \mathscr{V}[X, Y]]=f_{Z} \mathscr{V}[X, Y]
$$

for any local basic vector field $Z$ and for some function $f_{Z}$ depending on $Z$. 
Then, either

(i) $\mathscr{V}[X, Y]$ vanishes identically on $M$, so $\mathbf{H}$ is integrable and $\mathbf{H}$ is a foliation whose leaves are minimal surfaces in $M$ or

(ii) $\mathscr{V}[X, Y]$ never vanishes on $M$ and $\mathbf{H}$ is a contact structure.

(d) If, in addition to (a), (b), and (c), $M$ has a finite-fundamental group, then $\mathscr{V}[X, Y]$ never vanishes on $M$ and $\mathbf{H}$ is always a contact structure.

Conversely, if conclusion (i) above is obtained, then conditions (a), (b), and (c) necessarily hold on any closed, connected, oriented $C^{\infty}$ Riemannian 3-manifold $(M, g)$ with tangentially oriented flow $\mathbf{F}$.

REMARKS ON CONDITIONS (a), (b), AND (c) AND THE PROOF OF THE THEOREM. The Rummler-Sullivan criterion (see [9, 14]) gives a sufficient condition for a foliation $\mathbf{H}$ of a manifold of arbitrary dimension to be a foliation with the property that its leaves are minimal submanifolds. The above result does not require the two-dimensional distribution $\mathbf{H}$ to be integrable on $M^{3}$, but guarantees it unless $\mathbf{H}$ is a contact structure.

Condition (a) guarantees that, for basic vector fields $\{X, Y\}$ above, $\mathscr{V}[X, Y]$ is globally defined on $M$ and, simultaneously in the foliation case, that the leaves of $\mathbf{H}$ are minimal surfaces of $M$. If the flow $\mathbf{F}$ is bundle-like with respect to the metric $g$, then condition (a) is automatically satisfied since the metric restricted to $\mathbf{H}$ (and hence the transverse volume element) is (locally) projectible. That, under the stated conditions, $\mathscr{V}[X, Y]$ is globally defined (independent of the particular pair $\{X, Y\}$ selected above) was already observed in [1] (see also [2]).

Condition (b) is necessary to guarantee (6) and hence $V a \equiv 0$ on $M$.

In addition to the remarks about condition (c) at the end of the theorem, the following observation is in order. Suppose that conditions (a), (b) and conclusion (ii) of our theorem hold on a closed, connected, oriented, $C^{\infty}$ Riemannian 3-manifold $(M, g)$ with tangentially oriented flow $\mathbf{F}$. Then, (5) implies $a(x) \neq 0$ for any $x \in M$. Using the fact (see [2]) that $[Z, V]=\kappa(Z) V$ for any basic vector field $Z$, it is not hard to see that $[Z, a V]=(Z a / a+\kappa(Z)) a V=f_{Z} a V$. This is exactly condition (c). This is a somewhat surprising and pleasing development since, as noted in the theorem, condition (c) is also implied by conclusion (i) together with the other ancillary hypotheses. Condition (c) is also natural since it is well known that if $Z$ is basic and $W$ is vertical, then $[Z, W]$ is vertical.

The main ingredients of the proof are simple. By (5), the globally defined vector field $\mathscr{V}[X, Y]=a V$ for some real-valued function $a$ on $M$. Equations (6) and (9) each gives rise to a homogeneous differential equation. In (6), $d a / d t \equiv 0$ in any plaque of an integral curve of $\mathbf{F}$ and hence on all of that integral curve. In (9), $d a / d t \equiv h a$ for some real-valued function $h$ along the horizontal lift of any differentiable curve in $f_{\alpha}\left(U_{\alpha}\right)$. We use these facts repeatedly to show that the set $S$, defined below, is open in $M$, since it is clearly closed.

PROOF. As in [2], if a foliation of codimension 2 admits a basic transverse volume form $\mu$, then $\mathscr{V}[X, Y]$ is independent of the pair of basic vector fields, 
satisfying the above normalizing condition and so is globally defined on $M$. Now, this means that, on $M$,

$$
\mathscr{V}[X, Y]=a V,
$$

where $a$ is some globally defined function on $M$ function and $V$ is the unit vector field tangent to F. Again by [2], for such a codimension 2 foliation, $\kappa$ basic implies $\kappa$ closed. By the appendix of [6] (see [2]), we have, on $M$,

$$
0=d \kappa(X, Y)=-g\left(D_{V} \mathscr{V}[X, Y], V\right)=-V a
$$

and so, one can conclude that $\mathscr{V}[X, Y]$ is a Killing vector field on each plaque of the flow F. Clearly, (6) implies that $V a \equiv 0$ on this integral curve and, indeed, on $M$. Now, condition (c) implies

$$
\begin{aligned}
g([Z, \mathscr{V}[X, Y]], V) & =g\left(D_{Z} \mathscr{V}[X, Y], V\right)-g\left(T_{\mathscr{V}[X, Y]} Z, V\right) \\
& =g\left(f_{Z} \mathscr{V}[X, Y], V\right),
\end{aligned}
$$

or using the properties of $T$,

$$
g\left(D_{Z} \mathscr{V}[X, Y], V\right)+g\left(Z, T_{V} \mathscr{V}[X, Y]\right)=g\left(f_{Z} \mathscr{V}[X, Y], V\right)
$$

This means that, wherever $Z$ is defined,

$$
0=Z a+a\left(\kappa(Z)-f_{Z}\right)
$$

since $g(V, V)=1$.

Let $S=\{q \in M: \mathscr{V}[X, Y](q)=0\}$. Clearly, $S$ is closed in $M$. Note that if $\mathscr{V}[X, Y](q)=0$, (i.e., $a(q)=0$ by $(5)$ ), then $\mathscr{V}[X, Y] \equiv 0$ on the integral curve $L$ of $\mathbf{F}$ containing $q$. This follows because, by (6), $V a \equiv 0$ implies that $a$ is constant on the integral curves of $\mathbf{F}$.

We must show that for any $q \in S, \mathcal{V}[X, Y] \equiv 0$ on some path-connected open set $U$ of $q$. Since $q \in U_{\alpha}$ for some $\alpha$, we can choose $U \subset U_{\alpha}$. Now, let $q^{\prime} \in U$. We must show $a\left(q^{\prime}\right)=0$. There exists a path $\sigma$ in $U$, so $\sigma(0)=q$ and $\sigma(1)=q^{\prime}$. Observe for each $t_{i} \in[0,1]$, there exists an $\epsilon_{i}>0$, so if $t \in\left(t_{i}-\epsilon_{i}, t_{i}+\epsilon_{i}\right)$, then $\sigma(t)$ can be reached by a horizontal path from $\sigma\left(t_{i}\right)$ to the plaque of $\sigma(t)$, followed by a path in that plaque to $\sigma(t)$ itself.

This is achieved as follows. For this $U \subset U_{\alpha}, \sigma\left(t_{i}\right) \in U$ and $f_{\alpha}(U) \subset \mathbb{R}^{2}$ is open. There is a path $\gamma_{i}^{*}$ in $f_{\alpha}(U)$, connecting $f_{\alpha}\left(\sigma\left(t_{i}\right)\right)$ to $f_{\alpha}(\sigma(t))$. Then, $\gamma_{i}$, the unique horizontal lift of $\gamma_{i}^{*}$ starting at $\sigma\left(t_{i}\right)$, gives the desired horizontal path. (Indeed, $\gamma_{i}^{*}$ might be chosen as $f_{\alpha}(\sigma(u))$ for $t_{i} \leq u \leq t$.) Clearly, the tangent vector to $\gamma_{i}$ can be extended to a basic vector field. If $0=a\left(\sigma\left(t_{i}\right)\right)=$ $a\left(\gamma_{i}\left(t_{i}\right)\right),(9)$ implies

$$
a\left(\gamma_{i}(t)\right)=0 .
$$


Since $a$ is constant on the integral curves of $\mathbf{F}$ and hence on the plaques of these curves,

$$
a(\sigma(t))=0
$$

Now $[0,1]$ is compact. A standard Lebesgue covering argument allows us to apply (10) and (11) $n$ times to obtain

$$
\begin{aligned}
0 & =a(q)=a\left(\sigma\left(t_{0}\right)\right) \\
& =a\left(\gamma_{0}\left(t_{0}\right)\right)=a\left(\gamma_{0}\left(t_{1}\right)\right) \\
& =a\left(\sigma\left(t_{1}\right)\right)=a\left(\gamma_{1}\left(t_{1}\right)\right) \\
& =a\left(\gamma_{1}\left(t_{2}\right)\right)=a\left(\sigma\left(t_{2}\right)\right)=\cdots \\
& =a\left(\sigma\left(t_{n-1}\right)\right)=a\left(\gamma_{n-1}\left(t_{n-1}\right)\right) \\
& =a\left(\gamma_{n-1}\left(t_{n}\right)\right)=a\left(\gamma_{n-1}(1)\right) \\
& =a(\sigma(1))=a\left(q^{\prime}\right) .
\end{aligned}
$$

This means that $a\left(q^{\prime}\right)=0$. Since $q^{\prime}$ was any point of $U$, this means that

$$
a \equiv 0 \quad \text { on all of } U \text {, }
$$

that is, $\mathscr{V}[X, Y]=a V$ vanishes on $U$. This means that $S$ is open. By connectedness, $S=M$. Thus, if $\mathscr{V}[X, Y]$ vanishes at one point of $M$, it vanishes on $M$ identically and $\mathbf{H}$ is integrable. This proves (i) and (ii) since (14) guarantees that the leaves of $\mathbf{H}$ in (i) are minimal.

Suppose that (d) holds. Then, (a), (b), and (c) hold. Suppose (i) of our theorem is obtained. Let $\left\{X^{\prime}, Y^{\prime}\right\}$ be an orthonormal pair of horizontal vector fieldswhich need not be basic, since we do not impose the bundle-like hypothesis on the metric $g$. Then the mean curvature one-form associated with the foliation $\mathbf{H}$ is given by

$$
\beta(E)=g\left(E, A_{X^{\prime}} X^{\prime}+A_{Y^{\prime}} Y^{\prime}\right)
$$

for any vector field $E$ on $M$. The fact that $\mu$ is basic implies that the form $\beta$ vanishes identically by [2, pages 50-51]. Hence, the leaves of $\mathbf{H}$ are minimal submanifolds of $M$. Clearly, the foliation $\mathbf{H}$ can be described as the nullity of the nonsingular one-form $\alpha(E)=g(E, V)$, where $V$ is as above. But then we have a contradiction of a result of Sullivan (see [15]) as formulated by Tondeur (see [17, pages 21-22] or [18, pages 13-14]). Sullivan's theorem says that a codimension 1 foliation, defined by a nonsingular one-form on a closed 3manifold $M$ with finite fundamental group, cannot be (geometrically) taut, that is, cannot admit a metric so that the leaves of $\mathbf{H}$ are minimal (see [7, Chapter 3]). Thus, $\mathscr{V}[X, Y]$ never vanishes and so $\alpha \wedge d \alpha$ never vanishes on $M$, as can be easily checked on the basis $\{V, X, Y\}$ with $\{X, Y\}$ basic, as above. 
If conclusion (i) of the theorem is obtained $(\mathbf{H}$ is integrable with minimal leaves), then (6) above implies that $\kappa$ is horizontally closed. Hence, condition (b) of the theorem is obtained. Since $\mathscr{V}[X, Y] \equiv 0$, condition (c) is immediate. Let $\mu$ be the transverse volume form of the flow $\mathbf{F}$. The fact that the leaves of $\mathbf{H}$ are minimal means that $\beta$, the mean curvature one-form of the foliation $\mathbf{H}$, vanishes indentically. Since $\mathbf{H}^{\perp}=\mathbf{V}$ is clearly integrable, [18, page 40, Proposition 4.30] applies and $\theta(W)(\mu)=0$ for any vertical $W$. Since $\mu$ is the volume form of $\mathbf{H}, i(W)(\mu)=0$ for any vertical $W$. This means that $\mu$ is basic, so (a) is obtained. The proof of the theorem is complete.

EXAMPLE 1. Let $T^{n}$ denote the torus of dimension $n$ with the flat metric. Then the product bundle $S^{1} \rightarrow T^{3}=S^{1} \times T^{2} \rightarrow T^{2}$ gives a simple example of conclusion (i) of our theorem. Each copy of the circle $S^{1}$ is totally geodesic. The bundle is a product and hence the submersion from $T^{3} \rightarrow T^{2}$ is bundle-like, so conditions (a), (b), and (c) clearly hold. Each leaf of $\mathbf{H}$ is a totally geodesic (and hence minimal) $T^{2}$. Note that $\pi_{1}\left(T^{3}\right)$ is infinite.

EXAMPLE 2 (see [3, Example 1]). Let $\mathbf{h}$ be the Heisenberg Lie algebra with orthonormal basis $X, Y, V$ and the relation $[X, Y]=V$. Using the usual LeviCivita formula for the covariant derivative of left-invariant vector fields, one sees that

$$
\begin{aligned}
& D_{X} X=D_{Y} Y=D_{V} V=0, \\
& D_{X} Y=-D_{Y} X=\left(\frac{1}{2}\right) V, \\
& D_{X} V=D_{V} X=-\left(\frac{1}{2}\right) Y, \\
& D_{Y} V=D_{V} Y=\left(\frac{1}{2}\right) X .
\end{aligned}
$$

Then $\kappa \equiv 0$. If $g$ denotes the left-invariant metric, it is clearly bundle-like with respect to the flow on $M$ induced by $V$. Because the metric is bundle-like, the flow $\mathbf{F}$ induced by $V$ admits a holonomy invariant transverse volume form $\mu$, so (a) of our theorem holds. Conditions (b) and (c) also hold. These same structures is obtained on a compact quotient $M$ (see [13]) of the Heisenberg Lie group $H$ by a discrete subgroup. Let $\alpha^{1}, \alpha^{2}$, and $\alpha^{3}$ be the one-forms dual to $X, Y$, and $V$, respectively. Then one checks easily that $\alpha^{3} \wedge d \alpha^{3}$ never vanishes on $M$ and so is a contact structure (compare [4] for such a contact structure).

EXAMPLE 3. Perhaps the most obvious example of a contact structure arises from the flow defining the Hopf fibration on a unit sphere $S^{3}$. Each integral curve is a copy of the unit circle $S^{1}$. The distribution $\mathbf{H}$ orthogonal to that tangent to the flow is well known to be nonintegrable and indeed, in the above terminology, $\mathscr{V}[X, Y]$ is never zero (because of the curvature equations of O’Neill) so $\mathbf{H}$ is a contact structure. 
CONCLUDING REMARKS. The theorem is motivated by the consideration of the work in [8] (see also [3]). It would not be surprising if a version of this result is known in the more restricted case, that the flow $\mathbf{F}$ is bundle-like with respect to the metric $\mathrm{g}$, although we have been unable to track down a precise reference other than [8] (see also [3]). If the flow $\mathbf{F}$ is assumed to be Riemannian [there exists a metric $g^{\prime}$ on $M$ with respect to which $\mathbf{F}$ is bundle-like], then a result of Dominguez (see [18, page 81]) guarantees the existence of a bundlelike metric $g$ so that $\kappa$ is basic. Then a result of Kamber-Tondeur guarantees that $\kappa$ is closed. Thus, conditions (a) and (b) are unnecessary in this case. One still, however, needs (c) of our theorem. In the presence of such a bundle-like $g$, the conclusion of our theorem in the foliation case (i) is stronger: the leaves of $\mathbf{H}$ are totally geodesic. Interesting results of [10] (see Theorems 2 and 3 of that paper) give sufficient conditions for a foliation of surfaces on a compact 3-manifold to be non-geodesible.

In [8] the bundle-like hypothesis as well as constant curvature restrictions are assumed. Because of the work in [2], the dimensional restriction, the theorem of Sullivan, and the assumption that $\mu$ is basic, these are not required here. I would like to add that much is known about contact manifolds. See for example, David Blair's fine book Riemannian Geometry of Contact and Symplectic Manifolds (Birkhäuser, 2001).

ACKNOwLEdgments. I would like to thank Grant Cairns, my friend and collaborator in two papers, who offered helpful criticism. I would also like to thank my topology teachers B. Melvin Kiernan, John E. Derwent, and Jim Stasheff as well as my colleagues Leonid Khinkis and Chris Kinsey. A question by Professor Dennis Sullivan proved helpful in the exposition. I would like to thank the referees for helpful comments.

\section{REFERENCES}

[1] G. Cairns, Feuilletages geodesibles, Ph.D. thesis, Université des Sciences et Techniques du Languedoc, Montpellier, 1987.

[2] G. Cairns and R. H. Escobales Jr., Further geometry of the mean curvature oneform and the normal plane field one-form on a foliated Riemannian manifold, J. Austral. Math. Soc. Ser. A 62 (1997), no. 1, 46-63.

[3] , Note on a theorem of Gromoll-Grove, Bull. Austral. Math. Soc. 55 (1997), no. $1,1-5$.

[4] S. S. Chern and R. S. Hamilton, On Riemannian metrics adapted to threedimensional contact manifolds. With an appendix by Alan Weinstein, Workshop, Bonn, 1984, Lecture Notes in Mathematics, vol. 1111, Springer, Berlin, 1985, pp. 279-308.

[5] R. H. Escobales Jr., The integrability tensor for bundle-like foliations, Trans. Amer. Math. Soc. 270 (1982), no. 1, 333-339.

[6] R. H. Escobales Jr. and P. E. Parker, Geometric consequences of the normal curvature cohomology class in umbilic foliations, Indiana Univ. Math. J. 37 (1988), no. 2, 389-408. 
[7] M. H. Freedman and F. Luo, Selected Applications of Geometry to Low-Dimensional Topology, University Lecture Series, vol. 1, American Mathematical Society, Rhode Island, 1989.

[8] D. Gromoll and K. Grove, One-dimensional metric foliations in constant curvature spaces, Differential Geometry and Complex Analysis, Springer, Berlin, 1985, pp. 165-168.

[9] A. Haefliger, Some remarks on foliations with minimal leaves, J. Differential Geom. 15 (1980), no. 2, 269-284.

[10] D. L. Johnson and L. B. Whitt, Totally geodesic foliations on 3-manifolds, Proc. Amer. Math. Soc. 76 (1979), no. 2, 355-357.

[11]__ Totally geodesic foliations, J. Differential Geom. 15 (1980), no. 2, 225235.

[12] H. B. Lawson Jr., The Quantitative Theory of Foliations, CBMS Regional Conference Series in Mathematics, vol. 27, American Mathematical Society, Rhode Island, 1977.

[13] F. Raymond and A. T. Vasquez, 3-manifolds whose universal coverings are Lie groups, Topology Appl. 12 (1981), no. 2, 161-179.

[14] P. A. Schweitzer, Existence of codimension one foliations with minimal leaves, Ann. Global Anal. Geom. 9 (1991), no. 1, 77-81.

[15] D. Sullivan, A homological characterization of foliations consisting of minimal surfaces, Comment. Math. Helv. 54 (1979), no. 2, 218-223.

[16] Ph. Tondeur, Foliations on Riemannian Manifolds, Universitext, Springer-Verlag, New York, 1988.

[17] _ Geometry of Riemannian Foliations, Seminar on Mathematical Sciences, vol. 20, Department of Mathematics, Keio University, Yokohama, 1994.

[18]_, Geometry of Foliations, Monographs in Mathematics, vol. 90, Birkhäuser Verlag, Basel, 1997.

Richard H. Escobales Jr.: Department of Mathematics and Statistics, Canisius College, Buffalo, NY 14208, USA

E-mail address: escoba7r@canisius.edu 


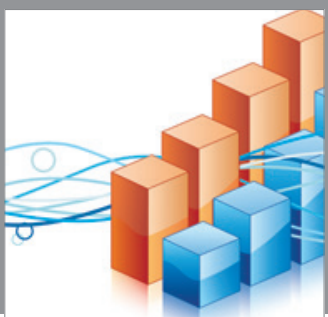

Advances in

Operations Research

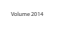

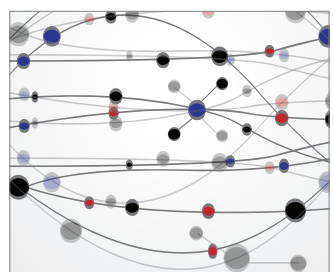

\section{The Scientific} World Journal
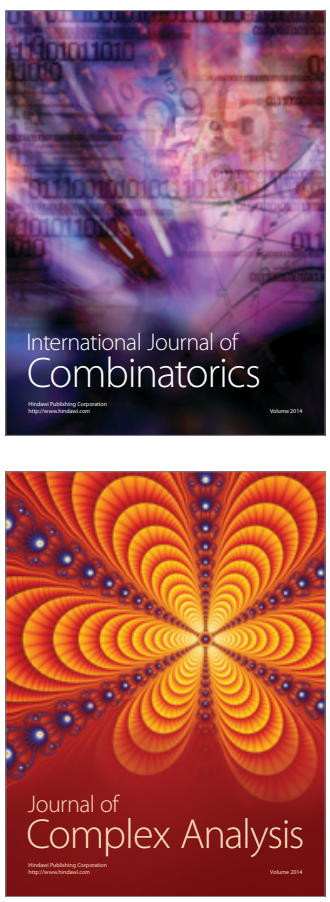

International Journal of

Mathematics and

Mathematical

Sciences
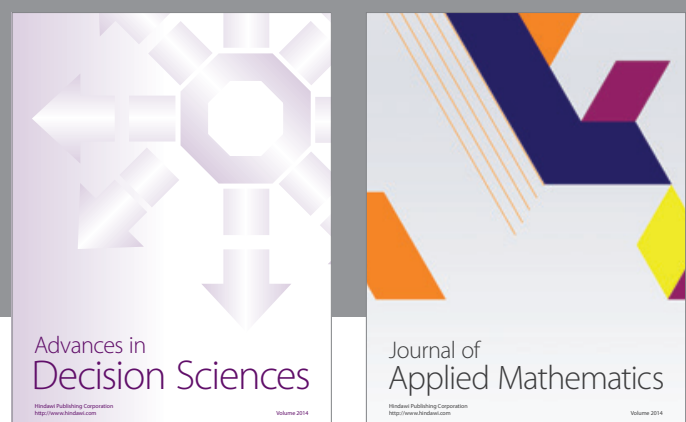

Journal of

Applied Mathematics
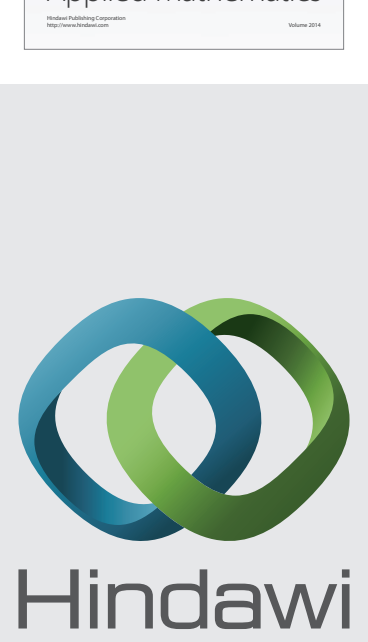

Submit your manuscripts at http://www.hindawi.com
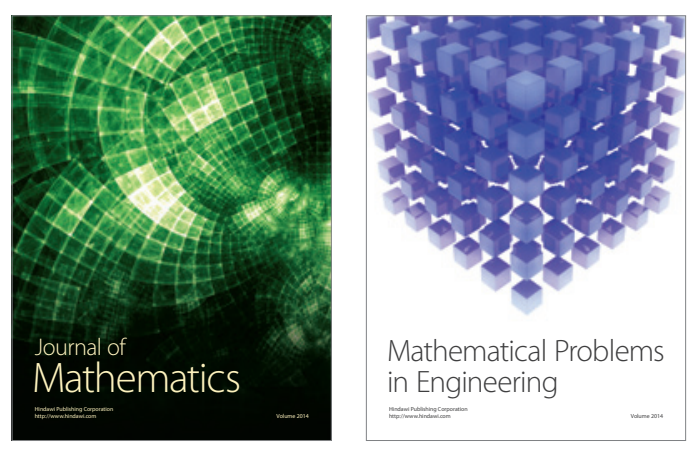

Mathematical Problems in Engineering
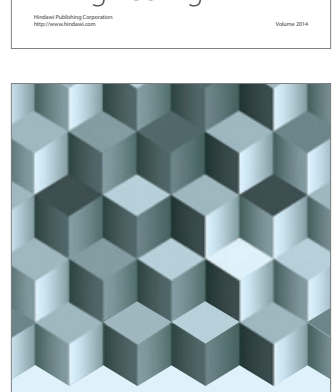

Journal of

Function Spaces
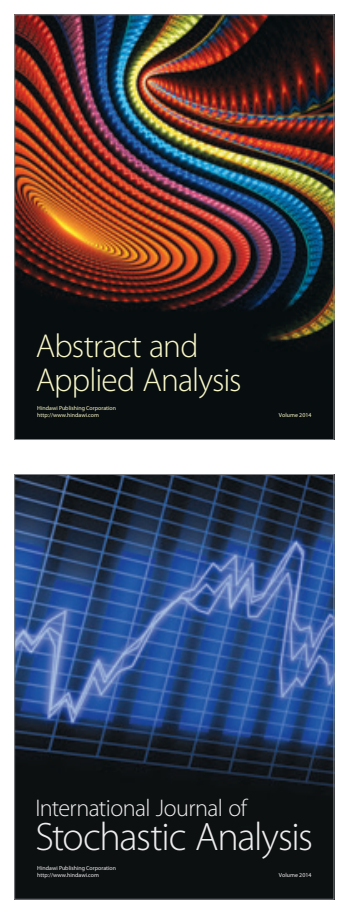

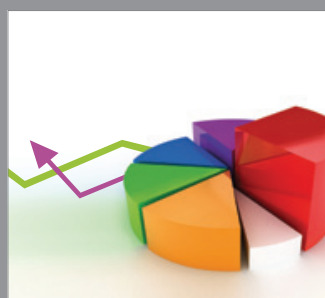

ournal of

Probability and Statistics

Promensencen
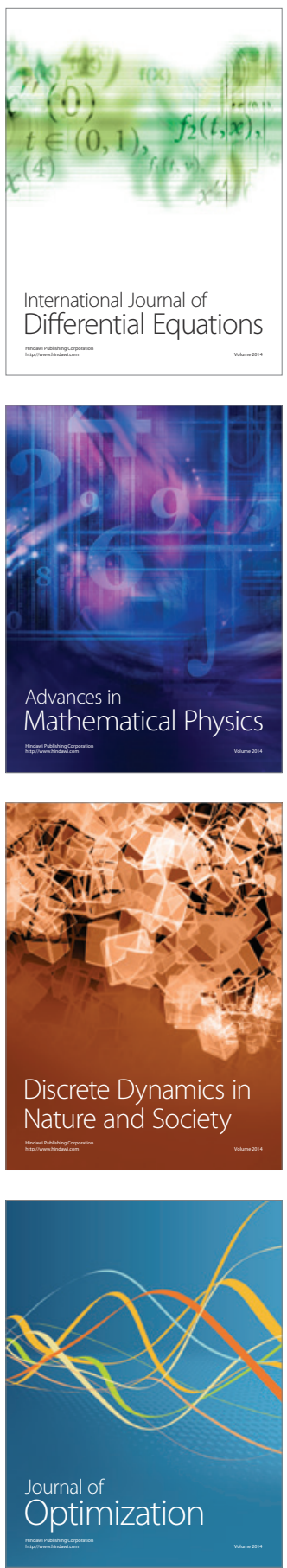